\title{
Fragile-X: Neuropsychological Test Performance, CGG Triplet Repeat Lengths, and Hippocampal Volumes
}

\author{
Pekka Jäkälä, ${ }^{\star}$ Tuomo Hänninen, ${ }^{\star}$ Markku Ryynänen, ${ }^{\S}$ Mikko Laakso, ${ }^{\star}$ Kaarina Partanen, ${ }^{\ddagger}$ Arto Mannermaa, ${ }^{\S}$ \\ and Hilkka Soininen* \\ *Department of Neuroscience and Neurology, ${ }^{\ddagger}$ Department of Radiology, and ${ }^{\S}$ Unit of Clinical Genetics of the Department of Gynecology \\ and Obstetrics, University Hospital and University of Kuopio, Finland
}

\begin{abstract}
We compared cognitive performance and hippocampal volumes using magnetic resonance imaging (MRI) in adult fragile-X $[\mathrm{fra}(\mathrm{X})]$ males and females with either premutation (pM) or full mutation (fM) $(n=10$ in all groups). Cognitive performance of $\mathrm{fM}$ males in the Wechsler Adult Intelligence Scale-Revised was worse than that of $\mathrm{pM}$ males, and the deficits in $\mathrm{fM}$ females were qualitatively similar, but less severe. In a visual memory test, both $\mathrm{fM}$ groups were impaired. In a list learning test, $\mathrm{fM}$ males were impaired in the learning phase and in delayed recognition. In a logical memory test, fM males and females were not significantly different from pM subjects. Hippocampal volumes normalized for intracranial or brain area did not significantly differ between $\mathrm{fM}$ and $\mathrm{pM}$ groups. However, positive correlations between left normalized hippocampal volumes and performance in many delayed memory tests observed in pM subjects were absent in fM subjects. Furthermore, in $>\mathbf{5 0 \%}$ of the fM subjects, nonspecific changes, such as enlargement of ventricles and perivascular spaces, focal hyperintensities in temporal pole white matter, and/or subjectively assessed atypical appearance of hippocampal morphology, were observed in MRI. The data suggest minor abnormalities in temporal lobe structures in adult fra(X) subjects with $\mathrm{fM}$. $(J$. Clin. Invest. 1997. 100:331-338.) Key words: fragile-X syndrome pathology $\bullet$ temporal lobe pathology $\bullet$ cognition $\bullet$ neuropsychology
\end{abstract}

\section{Introduction}

Fragile-X $[\mathrm{fra}(\mathrm{X})]^{1}$ syndrome is the most common form of inherited mental retardation, with an estimated incidence of 1 in

\section{Address correspondence to Dr. Pekka Jäkälä, Department of Neuro- science and Neurology, University Hospital and University of Kuo- pio, P.O. Box 1627, FIN-70211 Kuopio, Finland. Phone: 358-17- 163446; FAX: 358-17-162048; E-mail: pekka.jakala@uku.fi \\ Received for publication 14 February 1997 and accepted in revised form 21 April 1997.}

1. Abbreviations used in this paper: BA, brain area; CIA, coronal intracranial area; fM, full mutation; FMR1 gene, fragile-X mental retardation 1 gene; FMRP, FMR1 protein; fra(X), fragile-X; MMS, Mini-Mental Status examination; pM, premutation; MRI, magnetic resonance imaging; nBM, nucleus basalis magnocellularis; WAIS-R, Wechsler Adult Intelligence Scale—Revised.

J. Clin. Invest.

(C) The American Society for Clinical Investigation, Inc. 0021-9738/97/07/0331/08 \$2.00

Volume 100, Number 2, July 1997, 331-338
1,250 males and 1 in 2,000 females $(1,2)$. The syndrome is associated with an elongation of a small DNA fragment, containing a repeat of the trinucleotide CGG, located in the $5^{\prime}$ untranslated promoter region of the fragile- $\mathrm{X}$ mental retardation 1 (FMR1) gene in the X-chromosome. In normal individuals, the repetitive $(\mathrm{CGG})_{\mathrm{n}}$ sequence is stable with an average number of 5-35 repeats per individual. In fra $(\mathrm{X})$ premutation (pM), the CGG triplet repeat number is somewhere between 60 and 200 repeats, and the instability is emphasized as the number of repeats is increased. When 200 or more CGG repeats are present, the expanded repeat sequence and an adjacent $\mathrm{CpG}$ island are usually hypermethylated, a phenomenon associated with transcriptional silencing of the gene. This is commonly referred to as the FMR1 full mutation (fM) (3-6). In $\mathrm{pM}$, where there is no hypermethylation within the promoter region, there is normal or almost normal phenotype, whereas $\mathrm{fM}$ is characterized by mental retardation in most males and in $\sim 55 \%$ of females $(3,7-9)$.

The function of the FMR1 gene is still unknown. Methylation of the $\mathrm{CpG}$ island is considered a marker for inactivation of the FMR1 gene, which may lead to the fra(X) phenotype (4, 6). Correspondingly, the fM is usually associated with a lack of FMR1 protein (FMRP) expression, whereas pM carriers might have normal FMRP expression. In neurons, the protein synthesis units of the cell, polyribosomes, are located near the synapse. Since FMRP binds to RNA and ribosomes, it has been suggested that FMRP might be involved in the control of translation of certain proteins near the synapse, which are vital for synaptic maturation and plasticity (10-13). In agreement with this, immature synapse morphology in fra $(X)$ has been reported (12).

In normal individuals, the FMRP is most abundant in neuronal cells, particularly those in the cerebellum and Purkinje cells, although it is diffusely present throughout the gray matter (14-16). During fetal development, the highest levels of FMR1 transcription occur in the cholinergic neurons of the nucleus basalis magnocellularis (nBM) and in the pyramidal neurons of the hippocampus (15). According to animal and human studies, both the hippocampus and $\mathrm{nBM}$ are considered to be components of those neuroanatomical pathways involved in learning, memory, attention, processing of polysensory information, and regulation of affect (17-20). The nBM is also the major source of the cholinergic innervation of cortical and limbic structures (21). The cognitive dysfunctions in fM males consist of deficits in visual short-term memory, visual-spatial abilities, visual-motor coordination, processing of sequential information, and executive function deficits in attention (5, $7-9,22)$. Although there is less information about $\mathrm{fM}$ females, they appear to suffer cognitive abnormalities which are similar in quality, but less severe than those seen in fM males (23-27). Accordingly, there is a possibility that the absence of FMRP in the $\mathrm{nBM}$ region and/or hippocampus could be involved in 
the neuropsychological difficulties experienced by fra(X) individuals.

Recent developments in imaging techniques have provided noninvasive methods with which to visualize the brain. These may be of help in understanding how neurobiological factors contribute to the fra $(\mathrm{X})$ syndrome. Magnetic resonance imaging (MRI) is the most accurate imaging method available. It provides excellent gray/white matter contrast, which when combined with the possibility to make multiple observations in many planes, permits detection of changes in small, irregularly shaped structures such as the hippocampus (28-30). Previously, the right and left hippocampal volumes were reported to be significantly larger in a group of young males and females (aged 6-27 yr) with fra(X) when compared with a group of age- and IQ-matched controls (31). These young fra(X) subjects also revealed an age-related increase in the volume of the hippocampus and an age-related decrease in the volume of the superior temporal gyrus. Therefore, there is evidence to implicate temporal lobe regions in the behavioral and cognitive abnormalities associated with $\mathrm{fra}(\mathrm{X})$ syndrome.

The aim of this study was to compare the neuropsychological deficiencies and hippocampal volumes in MRI in adult $\mathrm{fra}(\mathrm{X})$ subjects with either fM or pM. Our hypotheses were that $(a)$ adult $\mathrm{fM}$ males would have the most pronounced hippocampal volume changes and that these would correlate with the neuropsychological deficits; and that $(b)$ the hippocampal volume changes and neuropsychological deficits would correlate to the CGG triplet repeat length in the FMR1 gene.

\section{Methods}

Patients. A representative sample of males and females with either $\mathrm{fra}(\mathrm{X}) \mathrm{fM}$ or $\mathrm{pM}(n=10$ in all four study groups) were randomly selected from an east Finland population representing different pedigrees (32). The study was approved by the local Ethics Committee, and informed consent for participation in the study was obtained from each subject and a near relative. None of the subjects was institutionalized. All the subjects underwent an extensive evaluation: medical history; clinical, general, and neurological examination; Mini-Mental Status examination (MMS); activities of daily living assessed by the scale of Blessed (range 0-28, 0 representing unimpaired performance); Hamilton Psychiatric Rating Scale for Depression (range 0-64, 0 representing no depression); a neuropsychological test battery including subtests from Wechsler Adult Intelligence ScaleRevised (WAIS-R); and tests of logical and visual memory as well as a list learning task. Finally, hippocampal volumes in MRI were measured. Three fM males failed to complete the neuropsychological test battery and failed to stay motionless in the imager due to hyperactivity or severe dyskinesia. In addition, one $\mathrm{pM}$ male, one $\mathrm{pM}$ female, and one fM female failed to stay motionless in the imager. None of these three subjects differed neuropsychologically from the others in their respective groups (data not shown). 10/10 fM males and 3/10 fM females had some features of fra(X)-phenotype including prominent ears or jaw, long face, loose joints, macroorchidism, skin abnormalities, or minor limb abnormalities, whereas none of the pM subjects had any of these features. In clinical neurological examination, 10/10 $\mathrm{fM}$ males and 2/10 fM females had minor abnormalities in either motor coordination, visuomotor skills, diadochokinesis, or visuospatial orientation. One 16-yr-old fM male had had several partial secondarily generalized epileptic seizures. However, the patient had been seizure free for $5 \mathrm{yr}$, and without antiepileptic medication (carbamazepine) for $1 \mathrm{yr}$.

Analysis of the FMR1 CGG-repeat length. DNA was prepared from venous blood of the subjects by standard phenol-chloroform procedure. The PCR-based nonradioactive analysis of CGG-repeat region of FMR-1 gene was carried out as described by Brown et al. (33) with some modifications. The amplification reactions were performed using a $5^{\prime}$ primer (5'-GAC GGA GGC GCC GCT GCC AGG-3') and a $3^{\prime}$ primer (5'-GTG GGC TGC GGG CGC TCG AGG-3'). The reaction mixture was in a final volume of $10 \mathrm{ml}$ containing 5 pmol of each primer, $200 \mathrm{mM}$ each of dATP, dCTP, dTTP, and 7-deazadGTP, $10 \%$ DMSO, $0.75 \mathrm{mM} \mathrm{MgCl}, 0.25$ U AmpliTaq DNA polymerase (Perkin Elmer), $1 \times$ PCR Buffer II (Perkin Elmer), and 50-100 ng of genomic DNA. The amplification conditions were: the initial denaturation at $94^{\circ} \mathrm{C}$ for 2 min followed by 30 cycles with denaturation at $94^{\circ} \mathrm{C}$ for $1 \mathrm{~min}$, annealing at $62^{\circ} \mathrm{C}$ for $1 \mathrm{~min}$, and elongation at $72^{\circ} \mathrm{C}$ for $2 \mathrm{~min}$. The PCR products were detected by (CGG)5-chemiluminescence probe hybridization after denaturing gel electrophoresis (6\% polyacrylamide, $8.3 \mathrm{M}$ urea) and electroblotting. Probe, chemiluminescent spray, and hybridizing reagents were supplied as a kit (Quick-Light, FMC). Fragment sizes in CGG-repeats were determined by migration relative to previously measured CGG-repeats with lengths from 16 to 70 repeats. In cases where no fragments were seen in a sample of a male or only one fragment was seen in a sample of a female, Southern hybridization with probe StB 12.33 was carried out as previously described in Mannermaa et al. (34). The subject was considered as a being a carrier $(\mathrm{pM})$ with repeats ranging from 60 to 200. The Southern hybridization procedure to detect the FMR1mutations usually shows a series of bands, or a smear, within the full mutation size range when an affected subject is studied. This is probably due to the mitotic fragility of the large triplet repeat. Therefore, the exact measuring of the mutation size is difficult, and for $\mathrm{fM}>200$ repeats and methylation were used as criteria. In statistical analyses, the value of 200 repeats was used for $\mathrm{fM}$ subjects.

Neuropsychological tests. Cognitive performance was assessed by using the WAIS-R verbal and performance IQ scores $(35,36)$. The scores from individual subtests of WAIS-R (Information, Digit Span, Vocabulary, Arithmetic, Similarities, Picture Completion, Picture Arrangement, Block Design, Object Assembly, and Digit Symbol) were used for the assessment of different cognitive domains. To shorten the test session, the Comprehension subtest was not done. Memory was assessed by using three tests: the Logical Memory Test and the Russell's adaptation of the Visual Reproduction Test from the Wechsler's Memory Scale (37), and a ten-item version of the Buschke Selective Reminding Test (a list learning test) (38). In the Logical Memory Test, the scores were the number of details of a story spontaneously recalled immediately and after the delay. In the Visual Reproduction Test, the scores were the total number of details of geometrical figures reproduced immediately and after the delay. In the Buschke Selective Reminding Test, the scores recorded were the total recall in six trials and in delayed recall, spontaneous recall, and recognition (correct responses and false positives). For all the memory tests, the delayed recall was assessed after about an hour of unrelated testing.

MRI imaging technique. The subjects were scanned with a $1.5 \mathrm{~T}$ Magnetom (SP4000; Siemens Magneton, Erlangen, Germany) using a standard head coil and a tilted coronal 3D gradient echo sequence (magnetization prepared rapid gradient echo: time of repetition 10 $\mathrm{ms}$, time of echo $4 \mathrm{~ms}$, time of inversion $250 \mathrm{~ms}$, flip angle $12^{\circ}$, field of vision $250 \mathrm{~mm}$, matrix $256 \times 192,1$ acquisition) resulting in contiguous $\mathrm{T}_{1}$-weighted partitions with slice thickness of $2.0 \mathrm{~mm}$ oriented perpendicular to the long axis of the hippocampus. The boundaries of the hippocampus were outlined by a trackball driven cursor, and the number of voxels within the region was calculated by using an inhouse developed program for standard work console. The outlining of the boundaries always proceeded from anterior to posterior in a sequential fashion. The volumetric measurements were performed by a single rater (M. Laakso) blinded to the clinical data and diagnostic category of the study subjects. Standard anatomical atlases of the human brain $(39,40)$, as well as several previous articles $(28,29,41)$, were used as guidelines to determine the boundaries of the hippocampus in oblique coronal MRI sections. The hippocampus is considered to include the dentate gyrus, the hippocampus proper, and the subicular 


\begin{tabular}{|c|c|c|c|c|c|}
\hline & fM male & pM male & fM female & pM female & ANOVA (F) \\
\hline Number & 10 & 10 & 10 & 10 & \\
\hline Age (yr) & $29.1 \pm 11.0^{*}$ & $52.4 \pm 19.7$ & $34.2 \pm 15.8^{*}$ & $48.3 \pm 15.0$ & $5.4^{\ddagger}$ \\
\hline MMS (range 0-30) & $12.9 \pm 5.9 * 8$ & $28.3 \pm 1.3$ & $26.3 \pm 4.3^{*}$ & $29.3 \pm 0.7$ & $42.2^{\|}$ \\
\hline Blessed (range 10-28) & $15.4 \pm 2.6 * \S$ & $10.7 \pm 0.9$ & $11.0 \pm 1.2 *$ & $10.3 \pm 0.5$ & $23.7^{\|}$ \\
\hline Hamilton (range 0-64) & $8.9 \pm 7.3$ & $4.8 \pm 3.2$ & $7.5 \pm 5.1$ & $6.8 \pm 4.9$ & 1.3 \\
\hline Abnormalities in neurological examination & $10 / 10$ & $0 / 10$ & $2 / 10$ & $0 / 10$ & \\
\hline
\end{tabular}

ANOVA $[\mathrm{F}(3,40)$ for all other data except for MMS F $(3,39)]$ with age as a covariate was used for between-group comparisons. Overall group effects: ${ }^{\ddagger} P<0.01,{ }^{\|} P<0.001$. fM males or females vs. their $\mathrm{pM}$ control groups, respectively: ${ }^{*} P<0.05$. fM males vs. fM females: ${ }^{\S} P<0.05$.

complex. The rostral end of the hippocampus where it first appears below the amygdala was the anatomical starting point. The caudal end of the hippocampus was taken as the section in which the crura of fornices depart from the hippocampal tail. To exclude the effect of individual brain and/or head size, the volumes were normalized for $(a)$ brain area (BA), which refers to the area of the brain measured at the level of the anterior commissure with the ventricular spaces excluded, and for $(b)$ coronal intracranial area (CIA) measured at the level of the anterior commissure. The intrarater and interrater agreements and reproducibility of this method have been reported previously (30, $42,43)$. The normalized values were used in the statistical analyses. Normalized hippocampal volumes of $\mathrm{fra}(\mathrm{X})$ subjects were also compared to those of age- and sex-matched healthy controls $(n=10$ in all control groups) derived from normal control material of Kuopio University Hospital (43). Finally, all scans were also evaluated by an experienced neuroradiologist (K. Partanen) who was blinded to the diagnostic category of the study subjects.

Statistical analyses. The data were analyzed by using Statistical Package for Social Sciences/Personal Computer (SPSS/PC+; SPSS Inc., Chicago, IL) V3.1 software. fM males and females were younger than their pM controls. Therefore, in comparisons of the means over the study groups, we used ANOVA adjusted for age. Normalized hippocampal volumes of age- and sex-matched healthy controls were compared to those of $\operatorname{fra}(\mathrm{X})$ subjects by one-way ANOVA followed by post-hoc Duncan's test. Correlations between normalized hippocampal volumes and neuropsychological tests were calculated by Pearson's correlation test, and correlations between CGG triplet repeat lengths and MRI data as well as neuropsychological tests were calculated by the partial correlation test, controlling for sex. In fM subjects, a CGG triplet repeat value of 200 was used in statistical analysis. The data are expressed as means $\pm \mathrm{SD}$. Values with $P<0.05$ were considered significant.

\section{Results}

The age, MMS, and Blessed and Hamilton scale scores of the study subjects are shown in Table I.

Cognitive performance in WAIS-R (Table II). fM males were inferior to $\mathrm{pM}$ males in all the subtests of WAIS-R, and had lower verbal and performance IQs than pM males. fM females were inferior to $\mathrm{pM}$ females in almost all the WAIS-R subtests, and the verbal and performance IQs of fM females were lower than those of $\mathrm{pM}$ females. When compared with $\mathrm{fM}$ females, fM males were inferior in all the subtests of the verbal part, and in almost all the subtests of the performance part of WAIS-R. The verbal and performance IQs of fM males were lower than those of $\mathrm{fM}$ females.

Memory tests (Table III). In the Logical Memory Test, fM males tended to recall less than $\mathrm{pM}$ males, and recalled signifi-

Table II. WAIS-R in fra $(X)$ Subjects

\begin{tabular}{|c|c|c|c|c|c|c|}
\hline Test & Possible range & fM male & pM male & fM female & pM female & ANOVA (F) \\
\hline \multicolumn{7}{|l|}{ Verbal part } \\
\hline Information & $0-32$ & $2.3 \pm 3.0 * \frac{1}{+}$ & $22.6 \pm 5.8$ & $10.9 \pm 5.1^{*}$ & $18.5 \pm 4.0$ & $20.0^{\S}$ \\
\hline Digit Span Forward & $0-14$ & $1.1 \pm 1.8^{* \leftarrow}$ & $6.1 \pm 1.6$ & $4.7 \pm 1.8$ & $6.2 \pm 1.6$ & $12.0^{\S}$ \\
\hline Digit Span Backward & $0-14$ & $0.6 \pm 0.5^{* \div+}$ & $5.4 \pm 2.0$ & $3.6 \pm 2.3^{*}$ & $5.7 \pm 1.6$ & $11.6^{\S}$ \\
\hline Vocabulary & $0-68$ & $7.4 \pm 5.0^{* \leftarrow \grave{T}}$ & $43.2 \pm 11.3$ & $25.8 \pm 15.9 *$ & $41.8 \pm 10.0$ & $22.9^{\S}$ \\
\hline Arithmetic & $0-25$ & $0.6 \pm 0.5^{* \dot{亠}}$ & $14.2 \pm 4.7$ & $5.0 \pm 3.9 *$ & $12.5 \pm 4.6$ & $17.8^{\S}$ \\
\hline Similarities & $0-34$ & $3.6 \pm 3.6^{* \div}$ & $24.7 \pm 5.9$ & $16.1 \pm 9.8^{*}$ & $24.8 \pm 4.7$ & $30.1^{\S}$ \\
\hline Verbal IQ & & $55.4 \pm 8.7^{* \dot{亠}}$ & $98.8 \pm 14.6$ & $71.8 \pm 17.6^{*}$ & $94.9 \pm 12.6$ & $20.6^{\S}$ \\
\hline \multicolumn{7}{|l|}{ Performance part } \\
\hline Picture Completion & $0-22$ & $6.3 \pm 4.4^{* \leftarrow+}$ & $14.9 \pm 3.2$ & $10.1 \pm 4.6^{*}$ & $12.5 \pm 2.4$ & $13.6^{\S}$ \\
\hline Picture Arrangement & $0-20$ & 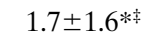 & $8.5 \pm 4.5$ & $7.3 \pm 6.5$ & $7.3 \pm 3.6$ & $7.8^{\S}$ \\
\hline Block Design & $0-51$ & $3.3 \pm 4.0 *$ & $25.4 \pm 12.2$ & $11.1 \pm 10.8 *$ & $23.0 \pm 7.0$ & $14.4^{\S}$ \\
\hline Object Assembly & $0-41$ & $9.0 \pm 6.4^{*}$ & $26.9 \pm 6.3$ & $15.3 \pm 8.1^{*}$ & $24.5 \pm 7.1$ & $10.1^{\S}$ \\
\hline Digit Symbol & $0-93$ & $11.0 \pm 9.1 * \frac{1}{+}$ & $37.6 \pm 14.8$ & $37.6 \pm 22.5$ & $43.5 \pm 13.3$ & $15.5^{\S}$ \\
\hline Performance IQ & & $54.1 \pm 7.8^{* \grave{!}}$ & $90.4 \pm 21.7$ & $73.0 \pm 15.8 *$ & $90.4 \pm 10.7$ & $8.0^{\S}$ \\
\hline
\end{tabular}

ANOVA $[\mathrm{F}(3,35)$ for all the data except for Block Design and Object Assembly $\mathrm{F}(3,34)]$ with age as a covariate was used for between-group comparisons. Overall group effects: ${ }^{\S} P<0.01$. fM males or females vs. their $\mathrm{pM}$ control groups, respectively: ${ }^{*} P<0.05$. fM males vs. $\mathrm{fM}$ females: ${ }^{*} P<0.05$. 
Table III. Memory Test Performance in fra $(X)$ Subjects

\begin{tabular}{|c|c|c|c|c|c|c|}
\hline Test & Possible range & fM male & pM male & fM female & pM female & ANOVA (F) \\
\hline \multicolumn{7}{|l|}{ Logical memory } \\
\hline Immediate recall & $0-21$ & $5.7 \pm 5.3^{* \grave{亠}}$ & $9.9 \pm 4.0$ & $10.4 \pm 4.6$ & $12.9 \pm 2.7$ & $5.6^{\S}$ \\
\hline Delayed recall & $0-21$ & $3.7 \pm 5.0^{* \frac{1}{+}}$ & $8.8 \pm 4.5$ & $8.7 \pm 5.7$ & $10.9 \pm 2.7$ & $5.2^{\S}$ \\
\hline \multicolumn{7}{|l|}{ Visual memory } \\
\hline Immediate recall & $0-21$ & $3.6 \pm 2.6^{* \ddagger}$ & $12.7 \pm 3.7$ & $7.9 \pm 3.7 *$ & $11.6 \pm 2.6$ & $17.8^{\S}$ \\
\hline Delayed recall & $0-21$ & $3.0 \pm 2.8^{*}$ & $11.9 \pm 4.2$ & $6.1 \pm 4.1 *$ & $9.6 \pm 4.3$ & $11.6^{8}$ \\
\hline \multicolumn{7}{|l|}{ List learning test } \\
\hline Total score & $0-60$ & $32.3 \pm 17.0^{*}$ & $44.8 \pm 6.3$ & $44.8 \pm 8.9$ & $51.7 \pm 3.7$ & $7.5^{\S}$ \\
\hline Long-term memory & $0-60$ & $26.9 \pm 21.1^{*}$ & $39.0 \pm 10.0$ & $41.6 \pm 11.0$ & $49.3 \pm 6.3$ & $6.7^{\S}$ \\
\hline Delayed recall & $0-10$ & $6.3 \pm 3.6$ & $7.7 \pm 1.5$ & $8.6 \pm 1.8$ & $9.1 \pm 1.1$ & $4.2^{\|}$ \\
\hline Recognition: correct responses & $0-10$ & $10.0 \pm 0.0$ & $9.5 \pm 1.0$ & $9.9 \pm 0.3$ & $10.0 \pm 0.0$ & 1.3 \\
\hline Recognition: false positives & $0-10$ & $4.7 \pm 4.7^{* \frac{亠}{\ddagger}}$ & $0.6 \pm 1.0$ & $0.6 \pm 1.9$ & $0.1 \pm 0.3$ & $6.0^{\S}$ \\
\hline
\end{tabular}

ANOVA $[\mathrm{F}(3,36)$ for all the data except for immediate recall in Logical Memory $\mathrm{F}(3,35)]$ with age as a covariate was used for between-group comparisons. Overall group effects: ${ }^{\S} P<0.01, \| P=0.01$. fM males or females vs. their pM control groups, respectively: ${ }^{*} P<0.05$. fM males vs. fM females: ${ }^{\ddagger} P<$ 0.05 .

cantly less than fM females, both immediately after story presentation and after a delay of $1 \mathrm{~h}$. $\mathrm{fM}$ and $\mathrm{pM}$ males and $\mathrm{fM}$ and $\mathrm{pM}$ females did not differ significantly in their performance in this test. In the Visual Reproduction Test, both fM males and females reproduced significantly fewer figure details than pM males and females, respectively, both immediately after figure presentation and after a delay of $1 \mathrm{~h}$. Furthermore, fM males reproduced significantly fewer figure details than fM females immediately after figure presentation. In the Selective Reminding list learning test, fM males learned fewer words during six trials (total recall) and recalled fewer words without reminding (long-term memory) than $\mathrm{pM}$ males during the learning phase. After a 1-h delay of unrelated testing, fM males recognized more false positive words from the list than $\mathrm{pM}$ males or $\mathrm{fM}$ females did. $\mathrm{fM}$ and $\mathrm{pM}$ females did not differ in this test.
MRI data (Table IV) and raw volumes. $\mathrm{fM}$ males tended to have larger BAs and CIAs than pM males, and significantly larger BAs and CIAs than fM females. $\mathrm{fM}$ females had larger BAs than pM females, whereas their CIAs did not differ. In general, males (fM and $\mathrm{pM})$ had significantly larger CIAs $(P<$ $0.02)$, and tended to have larger BAs $(P=0.07)$ than females (fM and $\mathrm{pM}$ ). After controlling for group, age correlated negatively with BAs $(r=-0.76, P<0.001)$, and tended to correlate negatively with CIAs $(r=-0.21, P>0.1)$. BAs and CIAs of $\mathrm{pM}$ and $\mathrm{fM}$ males and females did not differ significantly from those of age- and sex-matched controls.

The right and left hippocampal raw volumes of fM males tended to be larger than those of $\mathrm{pM}$ males, and were significantly larger than those of fM females. The hippocampal raw volumes of $\mathrm{fM}$ and $\mathrm{pM}$ females did not differ. In general, males (pM and $\mathrm{fM}$ ) had significantly larger right hippocampal

Table IV. Hippocampal Volumes in MRI in fra $(X)$ Subjects

\begin{tabular}{lcccc}
\hline Variable & fM Male & pM male & fM female & pM female \\
\hline BA $\left(\mathrm{mm}^{2}\right)$ & $9806 \pm 546^{*}$ & $8734 \pm 1055$ & $8697 \pm 821^{\ddagger}$ & $8532 \pm 1000$ \\
Controls & $9433 \pm 941$ & $9129 \pm 751$ & $8956 \pm 863$ & $8670 \pm 754$ \\
CIA $\left(\mathrm{mm}^{2}\right)$ & $13998 \pm 549^{*}$ & $13541 \pm 784$ & $13021 \pm 732$ & $13116 \pm 1022$ \\
Controls & $13250 \pm 716$ & $13081 \pm 729$ & $12463 \pm 757$ & $12412 \pm 723$ \\
HDX $\left(\mathrm{mm}^{3}\right)$ & $3736 \pm 281^{*}$ & $3298 \pm 499$ & $3133 \pm 378$ & $3179 \pm 450$ \\
Controls & $3690 \pm 638$ & $3855 \pm 460^{\S}$ & $3451 \pm 618$ & $3503 \pm 614$ \\
HSIN $\left(\mathrm{mm}^{3}\right)$ & $3668 \pm 325^{*}$ & $3085 \pm 475$ & $2949 \pm 461$ & $3041 \pm 557$ \\
Controls & $3478 \pm 466$ & $3609 \pm 450^{\S}$ & $3185 \pm 448$ & $3157 \pm 443$ \\
HDX:BA & $0.381 \pm 0.027$ & $0.380 \pm 0.051$ & $0.361 \pm 0.037$ & $0.374 \pm 0.042$ \\
Controls & $0.398 \pm 0.077$ & $0.424 \pm 0.046$ & $0.382 \pm 0.064$ & $0.442 \pm 0.050^{\S}$ \\
HDX:CIA & $0.267 \pm 0.020$ & $0.244 \pm 0.035$ & $0.241 \pm 0.031$ & $0.242 \pm 0.028$ \\
Controls & $0.278 \pm 0.043$ & $0.277 \pm 0.027$ & $0.274 \pm 0.038$ & $0.293 \pm 0.041^{\S}$ \\
HSIN:BA & $0.376 \pm 0.043$ & $0.353 \pm 0.033$ & $0.339 \pm 0.036$ & $0.356 \pm 0.043$ \\
Controls & $0.368 \pm 0.054$ & $0.413 \pm 0.065^{\S}$ & $0.352 \pm 0.043$ & $0.400 \pm 0.044$ \\
HSIN:CIA & $0.263 \pm 0.026$ & $0.228 \pm 0.029$ & $0.228 \pm 0.039$ & $0.232 \pm 0.038$ \\
Controls & $0.263 \pm 0.034$ & $0.269 \pm 0.036^{\S}$ & $0.250 \pm 0.020$ & $0.266 \pm 0.032^{\S}$ \\
& & & & 0.62 \\
\end{tabular}

Controls are age- and sex-matched healthy control subjects. ANOVA $[\mathrm{F}(3,34)$ for all the data) $]$ with age as a covariate was used for between $\mathrm{fM}$ and pM group comparisons, overall group effects: ${ }^{\|} P<0.05$. fM males or females vs. their pM control groups, respectively: ${ }^{\ddagger} P<0.05$. fM males vs. fM females: ${ }^{*} P<0.05$. Duncan's post-hoc analysis vs. age- and sex-matched healthy controls: ${ }^{\S} P<0.05$. $H D X$, right hippocampus; $H S I N$, left hippocampus. 


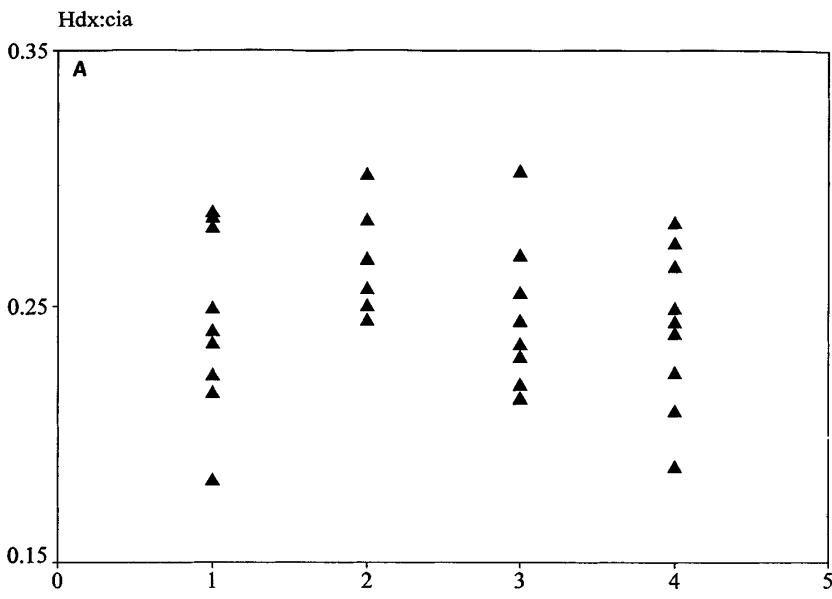

$1=\mathrm{pM}$ male $2=\mathrm{fM}$ male $3=\mathrm{pM}$ female $4=\mathrm{fM}$ female

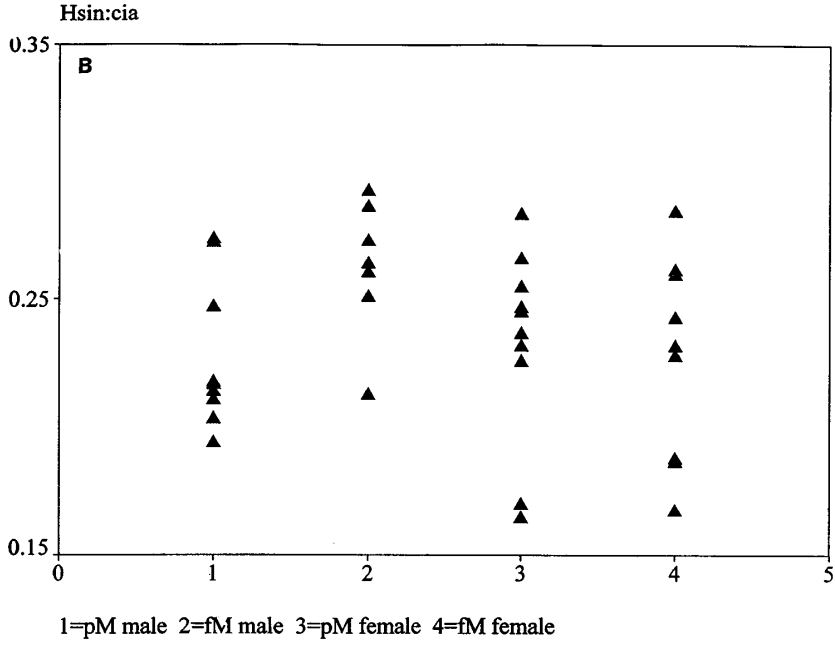

Figure 1. Right $(A)$ and left $(B)$ hippocampal volumes normalized for coronal intracranial area do not significantly differ between $\mathrm{fM}$ and $\mathrm{pM}$ males, $\mathrm{fM}$ and $\mathrm{pM}$ females, or $\mathrm{fM}$ males and females.

raw volumes $(P<0.05)$, and tended to have larger left hippocampal raw volumes $(P=0.05)$ than females ( $\mathrm{pM}$ and $\mathrm{fM})$. After controlling for age, right and left hippocampal raw volumes correlated positively with BAs $(r=0.58, P<0.001$ and $r=0.59, P<0.001$, respectively) and CIAs $(r=0.44, P<0.02$ and $r=0.36, P<0.05$, respectively). After controlling for group, age correlated negatively with both right $(r=-0.50$, $P<0.005)$ and left $(r=-0.63, P<0.001)$ hippocampal raw volumes. Right and left hippocampal raw volumes of $\mathrm{pM}$ males were smaller than those of their age- and sex-matched controls.

Normalized hippocampal volumes. Right and left hippocampal volumes normalized for either brain area (Table IV and Fig. 1) or coronal intracranial area (Table IV), did not differ between $\mathrm{fM}$ and $\mathrm{pM}$ males, $\mathrm{fM}$ and $\mathrm{pM}$ females, or fM males and females. On the other hand, pM females had smaller right hippocampal volumes normalized for either BA or CIA, and smaller left hippocampal volumes normalized for coronal intracranial area, than their age- and sex-matched controls. Furthermore, pM males had smaller left hippocampal volumes normalized for either BA or CIA than their age- and sex-matched controls.
Table V. Nonspecific MRI Changes in fra (X) Subjects

pM male fM male pM female fM female

Enlargement of perivascular spaces

Focal hyperintensities in temporal pole white matter Slightly atypical appearing hippocampal morphology Enlargement of ventricles Infarcts

$\begin{array}{llll}1 / 9 & 4 / 7 & 0 / 9 & 3 / 9 \\ 0 / 9 & 3 / 7 & 0 / 9 & 2 / 9 \\ 0 / 9 & 1 / 7 & 0 / 9 & 1 / 9 \\ 0 / 9 & 4 / 7 & 1 / 9 & 2 / 9 \\ 1 / 9 & 0 / 7 & 1 / 9 & 0 / 9\end{array}$

Nonspecific findings including subjective enlargement of ventricles and perivascular spaces, atrophy relative to age, white matter and perivascular changes in temporal poles, and subjectively atypical morphology of the hippocampi, were seen more often in fM subjects than in pM subjects (Table V). In addition to these changes, a 29-yr-old pM male had an old small contusional change in the right frontobasal region. The hippocampi of the fM male who had a history of partial secondary generalized seizures appeared normal.

Correlations between normalized hippocampal volumes, neuropsychology, and CGG triplet repeat lengths. Left and right hippocampal volumes normalized for BA and CIA were also analyzed for correlations between MMS and Blessed scale scores, the WAIS-R subtest scores, and the memory test scores in each group.

In $\mathrm{pM}$ males, left hippocampal volumes normalized for BA correlated positively with both immediate $(r=0.73, P<0.05)$ and delayed $(r=0.79, P<0.02)$ recall in the Logical Memory Test, and with Digit Span Forward $(r=0.74, P<0.05)$ in WAIS-R. Left hippocampal volumes normalized for CIA correlated positively with both immediate $(r=0.81, P<0.01)$ and delayed $(r=0.74, P<0.05)$ recall in the Visual Reproduction Test, total recall $(r=0.85, P<0.005)$, long-term memory $(r=$ $0.81, P<0.01)$, and delayed spontaneous recall $(r=0.72, P<$ $0.05)$ in the Selective Reminding list learning test, MMS test scores $(r=0.80, P<0.02)$, as well as Information $(r=0.68$, $P<0.05)$, Object Assembly $(r=0.67, P<0.05)$, Block Design $(r=0.78, P<0.02)$, and Digit Symbol $(r=0.75, P<0.05)$ in WAIS-R. Right hippocampal volumes normalized for CIA correlated positively with MMS test scores $(r=0.67, P<$ $0.05)$.

In pM females, left hippocampal volumes normalized for coronal intracranial area correlated positively with delayed recall in the Logical Memory Test $(r=0.63, P<0.05)$, total $(r=$ $0.66, P<0.05)$ and delayed recall $(r=0.69, P<0.05)$ in the Selective Reminding Test, as well as MMS test scores $(r=$ $0.65, P<0.05)$.

In $\mathrm{fM}$ males, left hippocampal volumes normalized for either BA or CIA correlated negatively with Similarities $(r=$ $-0.83, P<0.05$, and $r=-0.84, P<0.02$, respectively) and Picture Arrangement $(r=-0.86, P<0.02$, and $r=-0.82, P<$ 0.05 , respectively) subtests of WAIS-R.

In fM females, no significant correlations between normalized hippocampal volumes and any of the neuropsychological tests were found.

After controlling for sex, CGG triplet repeat lengths were 
analyzed for correlations between MRI data, MMS and Blessed scale scores, all the memory test scores, and verbal as well as performance IQs. The length of the CGG triplet repeat correlated positively with BA $(r=0.38, P<0.05)$ and Blessed scale scores $(r=0.50, P<0.01)$, and negatively with MMS test scores $(r=-0.52, P<0.01)$, verbal IQ $(r=-0.54, P<0.005)$, performance IQ $(r=-0.43, P<0.02)$, and immediate recall in the Visual Reproduction Test $(r=-0.47, P<0.02)$.

\section{Discussion}

In line with previous studies $(5,7-9,22,24,26)$, subjects with $\mathrm{fra}(\mathrm{X}) \mathrm{fM}$ had extensive and major cognitive deficits in WAIS-R when compared with $\mathrm{pM}$ subjects. $\mathrm{fM}$ males performed worse than pM males in all the subtests, and the deficits of fM females were qualitatively similar to those of fM males, although they were less severe. In the visual memory test, the abstract material to be remembered has to be drawn by the subject (37). Therefore, it is possible that the deficits in both immediate and delayed recall by both fM groups may relate more to deficits in visuoconstructive and/or visuospatial abilities (as indicated also by impaired performance by both fM groups in Block Design and Object Assembly in WAIS-R as compared to their pM counterparts), than to deficits in visual memory per se. However, in an earlier study (9), weaknesses in both fM males and females were found in short-term memory recall of visually presented abstract stimuli, whereas a consistent strength was observed for short-term memory recall of visually presented meaningful stimuli. Neither fM males nor fM females performed significantly worse than $\mathrm{pM}$ subjects in a logical memory test. In a list learning test, fM males were impaired in the learning phase and in delayed recognition, but not in delayed spontaneous recall. The lack of significant deficits in all the verbal memory measures in fM subjects when compared with pM subjects is partly in agreement with previous studies (5, 7-9), and suggests that fM subjects may have relative strengths in the domains of verbal memory or verbal functions when compared with their deficits in other cognitive functions. However, the small sample size in this study could also partially account for the failure to reach statistically significant differences in all the memory test variables between fM and $\mathrm{pM}$ groups.

The hippocampus is considered to belong to those neuroanatomical networks involved in learning, memory, attention, processing of polysensory information, and regulation of affect (17-20). These are all the domains of function which are often abnormal in subjects with fra(X) syndrome. Thus, hippocampal dysfunction and/or pathology could well account for some of the cognitive deficits observed in fra(X). Previously, the right and left raw hippocampal volumes were reported to be significantly larger in a group of young fra $(\mathrm{X})$ subjects when compared with controls (31). In this study in adult fra(X) subjects, we found no statistically significant differences in hippocampal volumes normalized for CIA or BA in fM subjects when compared with pM subjects. Furthermore, the normalized hippocampal volumes of $\mathrm{pM}$ subjects tended to be smaller, not larger, than those of their age- and sex-matched control subjects.

There are several methodological differences between the earlier study in young fra(X) subjects (31) and this study in adult fra $(\mathrm{X})$ subjects which may explain the conflicting results in hippocampal volumes between the studies. (a) The fra(X) group in the study of Reiss et al. (31) consisted of young (mean age $12.9 \pm 6.3 \mathrm{yr}$ ) subjects, whereas we compared adult subjects (the mean age in years being 29 in $\mathrm{fM}$ males, 52 in pM males, 34 in fM females, and 48 in pM females). (b) Reiss et al. (31) combined fra $(\mathrm{X})$ males and females into one group (six males and nine females), whereas in the present study fM males and $\mathrm{fM}$ females were kept as separate groups in the analyses. (c) Two of the fra $(\mathrm{X})$ subjects in the study by Reiss et al. (31) were mosaics and 13 had fM, whereas in the present study no mosaics were included. (d) The control group of Reiss et al. (31) consisted of subjects with different psychiatric diagnoses such as attention deficit hyperactivity disorder, depression, specific developmental disorder, oppositional defiant disorder, and autistic disorder, whereas we compared fM and pM groups between each other and vs. age- and sex-matched normal healthy control subjects. (e) In this study, we used hippocampal volumes normalized for either BA or CIA (see Methods), whereas Reiss et al. (31) used hippocampal raw volumes, although they had also ascertained that there were no differences in so-called "brain" values encompassing all the brain matter which was not included within the temporal lobes. It is also relevant to note that in this study, males ( $\mathrm{pM}$ and $\mathrm{fM}$ ) in general had larger CIAs, BAs, and hippocampal raw volumes than females (pM and $\mathrm{fM}$ ). Therefore, males and females (fM males and $\mathrm{fM}$ females and $\mathrm{pM}$ males and $\mathrm{pM}$ females) were not combined in the analysis. Furthermore, age-adjusted hippocampal raw volumes correlated significantly with BAs and CIAs. This indicates that the larger the BAs or CIAs of the individual adult fra $(\mathrm{X})$ subject, the larger one would expect the raw hippocampal volumes to be. However, after the hippocampal raw volumes were normalized for brain or head size, there were no differences between adult pM and fM subjects at the group level.

In the earlier study, an age-related increase in the volume of the hippocampus and an age-related decrease in the volume of the superior temporal gyrus was seen in young fra $(\mathrm{X})$ subjects (31). In contrast, in this study, age correlated negatively with hippocampal raw volumes and brain areas, i.e., the older the subjects, the smaller the hippocampal raw volumes and BAs. Thus, the increase in hippocampal raw volumes in young $\mathrm{fra}(\mathrm{X})$ subjects in the previous study could have been related to a growth and maturation process (31).

Although we found no significant changes in normalized hippocampal volumes between $\mathrm{fM}$ and $\mathrm{pM}$ groups, nonspecific changes were detected in the MRI investigation in $>50 \%$ of $\mathrm{fM}$ subjects. These changes included enlargement of ventricular and perivascular spaces, focal hyperintensities in temporal pole white matter, and/or somewhat atypical hippocampal morphology, indicative of structural abnormalities in temporal lobe structures in fM subjects.

Previously, the size of the hippocampus was also assessed in other genetic disorders using MRI. In Down's syndrome, MRI studies have reported hippocampal atrophy, this being more severe in demented patients. The same studies also reported an unexplained enlargement of the parahippocampal gyrus in Down's syndrome $(44,45)$. In Turner's syndrome, the size of the hippocampus has also been reported to be diminished (46).

The delayed memory test performance is traditionally viewed as a "measure of hippocampal function." Previously, in both Alzheimer's disease and temporal lobe epilepsy, associations between delayed recall performance in memory tests and 
hippocampal volumes detected by MRI have been noted (42, $43,47)$. For example, in Alzheimer patients, the volumes of the left hippocampus correlated significantly with immediate and delayed verbal memory; the smaller the volume, the more impaired the performance $(42,43)$. Furthermore, both animal and human studies have demonstrated that damage to the hippocampus and adjacent cortical areas causes profound learning and memory deficits, especially in delayed recall of information $(17,18)$. In this study, there were positive correlations between the left normalized hippocampal volumes and performance in many delayed verbal and visual memory tests and WAIS-R subtests in $\mathrm{pM}$ males and females, indicating that the performance was better in those with larger left normalized hippocampal volumes. It is tempting to speculate that the absence of these correlations in fM males and females could be a reflection of abnormalities in the function of hippocampal circuitries in fM subjects.

Our hypothesis was that the neuropsychological deficits and hippocampal volumes would correlate with the lengths of the CGG triplet repeats. Indeed, the length of the CGG triplet repeat correlated positively with brain area (see Methods) and Blessed scale scores, and negatively with MMS test scores, verbal and performance IQs, and immediate visual memory. These results suggest that those $\mathrm{fra}(\mathrm{X})$ patients with longer CGG triplet repeat lengths have larger brain areas, lower IQs, and poorer cognitive performance as measured by the MMS test, Blessed scale, or visual memory tests. However, we could not find any associations between normalized or raw hippocampal volumes and CGG triplet repeat lengths.

It should also be noted that the memory deficits associated with fra $(\mathrm{X})$ syndrome may not be due to hippocampal dysfunction and/or pathology alone, but could be related to pathology or abnormal function of other brain area(s) known to be important for learning and memory, e.g., diencephalic regions or frontal lobe structures, which were not measured in the present study. Furthermore, high levels of FMR1 transcription occur in the cholinergic neurons of the nBM (15). In Alzheimer's disease, the cholinergic cells of the basal forebrain, including nBM, degenerate and this cholinergic deafferentation correlates with clinical severity of the dementia (48). Therefore, the absence of FMRP in the nBM region and cholinergic dysfunction could also lead to the neuropsychological difficulties encountered by $\mathrm{fra}(\mathrm{X})$ patients. The posterior cerebellar vermis was reported previously to be hypoplastic in both fra $(\mathrm{X})$ males and females when compared with controls, and this was suggested to correlate with deficits in the processing or modulation of sensory stimuli, motor behavior, and language functions in $\mathrm{fra}(\mathrm{X})$ subjects $(49,50)$. The expression of the murine homologue of the FMR1 gene is most highly concentrated in the granular layers of the hippocampus and cerebellum, and expression of an FMR1/beta-galactosidase fusion gene in transgenic mice is most abundant in the hippocampus and granule cell layer of the cerebellum (16). Therefore, abnormal function of the hippocampus, nBM, or cerebellum could produce separate, perhaps dissociable, contributions to the neurobehavioral and neuropsychological disturbances experienced by individuals with fra $(\mathrm{X})$ syndrome. Finally, subjects with fra $(\mathrm{X})$ were reported to have an increased volume of the caudate nucleus, and, in males, also the lateral ventricle (51). Both caudate and lateral ventricular volumes correlated with IQ, and caudate volume correlated with methylation status of the FMR1 gene. Thus, caudate dysfunction could also contribute to the neuropsychological deficits seen in $\operatorname{fra}(\mathrm{X})$ syndrome.

In conclusion, adult fra $(\mathrm{X}) \mathrm{fM}$ subjects did not have significantly larger hippocampal volumes normalized for either BA or CIA than adult fra $(\mathrm{X}) \mathrm{pM}$ subjects. However, the absence of volumetric changes in MRI does not exclude the possibility that adult $\mathrm{fM}$ subjects may have neuropathological changes affecting the hippocampus. Indeed, the nonspecific MRI changes observed in $\mathrm{fM}$ subjects suggest that these subjects may have minor structural abnormalities in their hippocampi. In line with this, the positive correlations between left normalized hippocampal volumes and performance in delayed memory tests observed in pM subjects were absent in fM subjects. Recent developments in the functional imaging techniques (e.g., $\mathrm{T}_{2}$ relaxation times in MRI, MR spectroscopy, and functional MRI) may help to reveal abnormalities in hippocampal function in adult fra $(\mathrm{X})$ subjects with $\mathrm{fM}$. Furthermore, neuropathological investigations of the temporal lobe structures and characterization of FMRP expression in hippocampus and other areas of the brain in adult fra $(\mathrm{X})$ subjects will help to clarify the neurobiology of this tragic disorder.

\section{Acknowledgments}

We thank Mrs. Pirkko Jokela and Mrs. Inkeri Happonen for carrying out the DNA analysis of the CGG-repeats, research nurses Helena Luostarinen and Markku Kalinen for excellent technical assistance, and Dr. Ewen MacDonald for checking the language of the manuscript.

P. Jäkälä is supported by The Academy of Finland.

\section{References}

1. Snow, K., L.K. Doud, R. Hagerman, R.G. Pergolizzi, S.H. Erster, and S.N. Thibodeau. 1993. Analysis of a CGG sequence at the FMR1 locus in fragile X families and in the general population. Am. J. Hum. Genet. 53:1217-1228.

2. Webb, T. 1989. The epidemiology of the fragile $\mathrm{X}$ syndrome. In The Fragile X Syndrome. K.E. Davies, editor. Oxford University Press, Oxford, United Kingdom. 40-45.

3. Reiss, A.L., L.S. Freund, T.L. Baumgardner, M.T. Abrams, and M.B. Dencla. 1995. Contribution of the FMR1 gene mutation to human intellectual dysfunction. Nat. Genet. 11:331-334.

4. Hori, T., M. Yamauchi, N. Seki, S. Tsuji, and I. Kondo. 1993. Heritable unstable DNA sequences and hypermethylation associated with fragile $\mathrm{X}$ syndrome in Japanese families. Clin. Genet. 43:34-38.

5. Turk, J. 1992. The fragile-X syndrome, on the way to a behavioural phenotype. Br. J. Psychiatry. 160:24-35.

6. Rocchi, M., N. Archidiacono, A. Rinaldi, G. Filippi, G. Bartolucci, G.S. Fancello, and M. Siniscalco. 1990. Mental retardation in heterozygotes for the fragile-X mutation: evidence in favor of an $\mathrm{X}$ inactivation-dependent effect. Am. J. Hum. Genet. 46:738-743.

7. Sobesky, W.E., B.F. Pennington, D. Porter, C.E. Hull, and R.J. Hagerman. 1994. Emotional and neurocognitive deficits in fragile X. Am. J. Med. Genet. 51:378-385.

8. Cianchetti, C., G. Sannio-Fancello, A.-L. Fratta, F. Manconi, A. Orano, M.P. Pischedda, D. Pruna, G. Spinicci, N. Archidiacono, and G. Filippi. 1991 Neuropsychological, psychiatric, and physical manifestations in 149 members from 18 fragile X families. Am. J. Med. Genet. 40:234-243.

9. Freund, L.S., and A.L. Reiss. 1991. Cognitive profiles associated with the fra (X) syndrome in males and females. Am. J. Med. Genet. 38:542-547.

10. Verheij, C., C.E. Bakker, E. De Graaf, J. Keulemans, R. Willemsen, A.J. Verkerk, H. Galjaard, A.J. Reuser, A.T. Hoogeveen, and B.A. Oostra. 1993. Characterization and localization of the FMR1 gene product associated with fragile X syndrome. Nature (Lond.). 363:722-724.

11. Siomi, H., M.C. Siomi, R.L. Nussbaum, and G. Dreyfuss. 1993. The protein product of the fragile X gene, FMR1, has characteristics of an RNA-binding protein. Cell. 74:291-298.

12. Greenough, W., I.J. Weiler, J. Eberwine, T.A. Comery, S.A. Irwin, and A.D. Brazelton. 1996. Synaptic role of FMRP: what are the implications of synthesis of the protein at synapses. The 5th International Fragile X Conference, Portland, OR.

13. Weiler, I.J., A.D. Brazelton, and W.T. Greenough. 1996. Fragile X pro- 
tein binding to translation complexes. The 5th International Fragile X Conference, Portland, OR.

14. Hinds, H.L., C.T. Ashley, J.S. Sutcliffe, D.L. Nelson, S.T. Warren, D.E. Housman, and M. Schalling. 1993. Tissue specific expression of FMR1 provides evidence for a functional role in fragile X syndrome. Nat. Genet. 3:36-43.

15. Abitbol, M., C. Menini, A.-L. Delezoide, T. Rhyner, M. Vekemans, and J. Mallet. 1993. Nucleus basalis magnocellularis and hippocampus are the major sites of FMR1 expression in the human fetal brain. Nat. Genet. 4:147-153.

16. Hergersberg, M., K. Matsuo, M. Gassmann, W. Schaffner, B. Luscher, T. Rulicke, and A. Aguzzi. 1995. Tissue-specific expression of a FMR1/betagalactosidase fusion gene in transgenic mice. Hum. Mol. Genet. 4:359-366.

17. Murray, E.A., D. Gaffan, and M. Mishkin. 1993. Neural substrates of visual stimulus-stimulus association in rhesus monkeys. J. Neurosci. 13:45494561 .

18. Incisa-della-Rocchetta, A., D.G. Gadian, A. Connelly, C.E. Polkey, G.D. Jackson, K.E. Watkins, C.L. Johnson, M. Mishkin, and F. Vargha-Khadem. 1995. Verbal memory impairment after right temporal lobe surgery: role of contralateral damage as revealed by $1 \mathrm{H}$ magnetic resonance spectroscopy and T2 relaxometry. Neurology. 45:797-802.

19. Ojemann, G.A., O. Creutzfeldt, E. Lettich, and M.M. Haglund. 1988. Neuronal activity in human lateral temporal cortex related to short-term verbal memory, naming and reading. Brain. 111:1383-1403.

20. Creutzfeldt, O., G.A. Ojemann, and E. Lettich. 1989. Neuronal activity in the human lateral temporal lobe. Responses to speech. Exp. Brain Res. 77: 451-457.

21. Mesulam, M.M. 1995. Cholinergic pathways and the ascending reticular activating system of the human brain. Ann. NY Acad. Sci. 757:169-179.

22. Maes, B., J.-P. Fryns, M. Van Walleghem, and H. Van den Berghe. 1994. Cognitive functioning and information processing of adult mentally retarded men with fragile-X syndrome. Am. J. Med. Genet. 50:190-200.

23. Cronister, A., R. Schreiner, M. Wittenberger, K. Amiri, K. Harris, and R.J. Hagerman. 1991. Heterozygous fragile X female: historical, physical, cognitive, and cytogenetic features. Am. J. Med. Genet. 38:269-274.

24. Cronister, A., R.J. Hagerman, M. Wittenberger, and K. Amiri. 1991. Mental impairment in cytogenetically positive fragile X females. Am. J. Med. Genet. 38:503-504.

25. Brainard, S.S., R.A. Schreiner, and R.J. Hagerman. 1991. Cognitive profiles of the carrier fragile X woman. Am. J. Med. Genet. 38:505-508.

26. de von Flindt, R., B. Bybel, A.E. Chudley, and F. Lopes. 1991. Shortterm memory and cognitive variability in adult fragile $\mathrm{X}$ females. Am. J. Med. Genet. 38:488-492.

27. Mazzocco, M.M.M., R.J. Hagerman, A. Cronister-Silverman, and B.F. Pennington. 1991. Specific frontal lobe deficits among women with the fragile X gene. J. Am. Acad. Child Adolesc. Psychiatry. 31:1141-1148.

28. Watson, C., F. Andermann, P. Gloor, M. Jones-Gotman, T. Peters, A. Evans, A. Olivier, D. Melanson, and G. Leroux. 1992. Anatomical basis of amygdaloid and hippocampal volume measurement by magnetic resonance imaging. Neurology. 42:1743-1750.

29. Bartzokis, G., J. Mintz, P. Marx, D Osborn, D Gutkind, F Chiang, C.K. Phelan, and S.R. Marder. 1993. Reliability of in vivo measures of hippocampus and other brain structures using MRI. Magn. Reson. Imaging. 11:9931006.

30. Soininen, H., K. Partanen, A. Pitkänen, P. Vainio, T. Hänninen, M. Hallikainen, K. Koivisto, and P.J. Riekkinen, Sr. 1994. Volumetric MRI analysis of the amygdala and the hippocampus in subjects with age-associated memory impairment: correlation to visual and verbal memory. Neurology. 44:1660-1668.

31. Reiss, A.L., J. Lee, and L. Freund. 1994. Neuroanatomy of the fragile X syndrome: the temporal lobe. Neurology. 44:1317-1324.

32. Ryynänen, M., L. Pulkkinen, P. Kirkinen, and S. Saarikoski. 1994. Frag-
ile-X syndrome in east Finland: molecular approach to genetic and prenatal diagnosis. Am. J. Med. Genet. 51:463-465.

33. Brown, T., G. Houck, A. Jeziorowska, F.N. Levinso, X. Ding, C. Dobkin, N. Zhong, J. Henderson, S.S. Brooks, and E.C. Jenkins. 1993. Rapid fragile $\mathrm{x}$ carrier screening and prenatal diagnosis using nonradioactive PCR test. JAMA (J. Am. Med. Assoc.). 270:1569-1575.

34. Mannermaa, A., L. Pulkkinen, E. Kajanoja, M. Ryynänen, and S. Saarikoski. 1996. Deletion in the FMR1 gene in a Fragile-x-male. Am. J. Med. Genet. 64:293-295.

35. Wechsler, D. 1981. WAIS-R Manual. Psychological Corporation, New York

36. Wechsler, D. 1974. Wechsler Memory Scale Manual. Psychological Corporation, San Antonio, TX.

37. Russell, E.W. 1975. A multiple scoring method for the assessment of complex memory functions. J. Consult. Clin. Psychol. 43:800-809.

38. Buschke, H., and P.A. Fuld. 1974. Evaluating storage, retention, and retrieval in disordered memory and learning. Neurology. 24:1019-1025.

39. Duvernoy, H.M. 1988. The Human Hippocampus: An Atlas of Applied Anatomy. J.F. Bergman Verlag, Munich.

40. DeArmond, S.J., M.M. Fusco, and M.M. Dewey. 1989. Structure of the Human Brain. A Photographic Atlas, 3rd ed. Oxford University Press, New York/Oxford, United Kingdom.

41. Bronen, R.A., and G. Cheung. 1991. MRI of the temporal lobe: normal variations, with special reference toward epilepsy. Magn. Reson. Imaging. 9: 501-507.

42. Laakso, M.P., H. Soininen, K. Partanen, E.L. Helkala, P. Hartikainen, P. Vainio, M. Hallikainen, T. Hänninen, and P. R. Riekkinen, Sr. 1995. Volumes of hippocampus, amygdala, and frontal lobes in the MRI-based diagnosis of early Alzheimer's disease: correlation with memory functions. J. Neural Transm. 9:73-86.

43. Laakso, M. 1996. MRI of hippocampus in incipient Alzheimer's disease. Ph.D. thesis. Series of reports, Department of Neurology, University of Kuopio.

44. Kesslak, J.P., S.F. Nagata, I. Lott, and O. Nalcioglu. 1994. Magnetic resonance imaging analysis of age-related changes in the brains of individuals with Down's syndrome. Neurology. 44:1039-1045.

45. Raz, N., L.J. Torres, S.D. Briggs, W.D. Spencer, A.E. Thornton, W.J. Loken, F.M. Gunning, J.D. McQuain, N.R. Driesen, and J.D. Acker. 1995. Selective neuroanatomic abnormalities in Down's syndrome and their cognitive correlates: evidence from MRI morphometry. Neurology. 45:356-366.

46. Reiss, A.L., L. Freund, L. Plotnick, T. Baumgardner, K. Green, A.C. Sozer, M. Reader, C. Boehm, and M.B. Denckla. 1993. The effects of X monosomy on brain development: monozygotic twins discordant for Turner's syndrome. Ann. Neurol. 34:95-107.

47. Pitkänen, A., M. Laakso, R. Kälviäinen, K. Partanen, P. Vainio, M. Lehtovirta, P. Riekkinen, and H. Soininen. 1996. Severity of hippocampal atrophy correlates with the prolongation of MRI T2 relaxation time in temporal lobe epilepsy but not in Alzheimer's disease. Neurology. 46:1724-1730.

48. Soininen, H., K.J. Reinikainen, J. Partanen, E.L. Helkala, L. Paljärvi, and P.J. Riekkinen. 1992. Slowing of electroencephalogram and choline acetyl transferase activity in post mortem cortex in definite Alzheimer's disease. Neuroscience. 49:529-535.

49. Reiss, A.L., E. Aylward, L.S. Freund, P.K. Joshi, and N.B. Bryan. 1991 Neuroanatomy of fragile X syndrome: the posterior fossa. Ann. Neurol. 29:26-32.

50. Reiss, A.L., L. Freund, J.E. Tseng, and P.K. Joshi. 1991. Neuroanatomy in fragile X females: the posterior fossa. Am. J. Hum. Genet. 49:279-288.

51. Reiss, A.L., M.T. Abrams, R. Greenlaw, L. Freund, and M.B. Denckla 1995. Neurodevelopmental effects of the FMR-1 full mutation in humans. Nat. Med. 1:159-167. 